the sequence of the gene regions encoding the cleavage sites of the neuronal VAMPs of mouse (Mus musculus) and chicken (Gallus domesticus), species respectively highly and poorly sensitive to the neurotoxins ${ }^{7}$. Sequences were obtained by nested polymerase chain reaction (PCR) amplification and direct sequencing. Primers were designed from aligned VAMP sequences ${ }^{1,4,6}$ and some of them were isoform-specific; either genomic DNA (mouse) or brain complementary DNA (chicken) were used as templates for PCR amplification. Comparison of the partial VAMP sequences (see figure) shows the occurrence of substitutions. VAMP-1 and VAMP-2 nucleotide changes are all synonymous except for the replacement of a Val for a Gln residue on VAMP-1 at the site of neurotoxin proteolytic cleavage in the two toxin-resistant species (rat and chicken).

These results support the idea that the low toxin sensitivity of rat and chicken may be due, at least partially, to a mutation that modifies the toxin cleavage site. From an evolutionary point of view, the triplet change at the site of toxin action in rat and chicken can be considered an example of convergent molecular evolution driven by the toxin, which acts as a selective agent.

\section{Tomaso Patarnello}

Luca Bargelloni

Dipartimento di Biologia,

Ornella Rossetto

Giampietro Schiavo

Cesare Montecucco

Dipartimento di Scienze Biomediche,

Università di Padova,

Via Trieste 75 ,

35121 Padova, Italy

1. Trimble, W.S., Cowan, D. M. \& Scheller, R. H. Proc. natn Acad. Sci. U.S.A. 85, 4538-4542 (1988)

2. Sollner, T. etal. Nature 362, 318-324 (1993)

3. Warren, G. B. Nature 362, 297-298(1993)

4. Elferink, L. A., Trimble, W. S. \& Scheller, R. H. J. biol. Chem. 264, 11061-11064 (1989).

5. Schiavo, G. et al. Nature 359, 832-835 (1992).

6. Archer, B. T. etal.J. biol. Chem. 265, 17267-17273 (1990).

7. Payling Wright, G. Pharmac. Rev. 7, 413-465 (1955)

SIR - Recent discoveries have led to the realization that proteins involved in synaptic transmission have counterparts in the normal secretory pathways of animal cells and yeast, and thus that the basic machinery involved in these disparate processes is similar ${ }^{1,2}$. Synaptic proteins with homologues elsewhere include rab3a, the syntaxins and the VAMPs/ synaptobrevins. I have found an additional similarity between a protein implicated in neurotransmitter release and a yeast $S E C$ gene product, which appears previously to have gone unnoticed.

Mutations in the unc-18 gene of the nematode Caenorhabditis elegans result in severe paralysis, and confer partial resistance to an acetylcholinesterase inhibitor. The affected animals accumulate higher

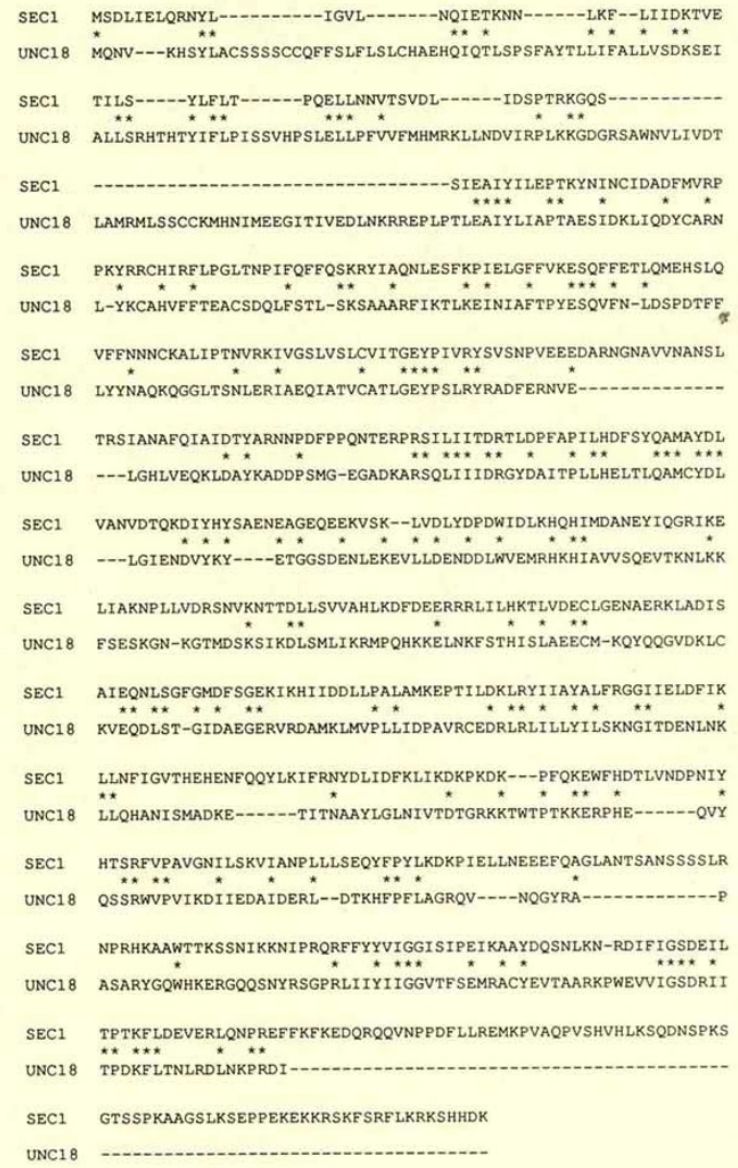

Alignment of the sequences of yeast Sec1 protein and the C. elegans unc-18 product. Twenty per cent of the residues are identical, and are indicated by asterisks; similarities, which are common, are not marked.

than normal levels of acetylcholine, although they have normal levels of cholinesterase activity ${ }^{3}$. By electron microscopy, the cholinergic synapses of an animal homozygous for the e81 allele (in which the unc-18 transcript is greatly reduced or absent ${ }^{3}$ ) appear to be largely normal in structure, and they contain normal numbers of synaptic vesicles (J. White, personal communication). A possible explanation of this phenotype is that the primary defect in unc-18 lies in the fusion of acetylcholinecontaining vesicles with the plasma membrane. Vesicle components are normally recycled from the plasma membrane; inefficient fusion would interrupt this cycle but lead to no net change in vesicle number. It would, however, inhibit release of the neurotransmitter and its subsequent degradation by extracellular cholinesterase.

A database search using the TBLASTN program $^{4}$ revealed that the unc- 18 prod-

\footnotetext{
1. Soliner, T. etal. Nature 362, 318-324 (1993)

2. Barinaga, M. Science 260, 487-489 (1993).

2. Barinaga, M. Science 260, 487-489 (1993).
3. Hosong, R. et al. J. Neurochem. 58, 1517-1525 (1992)

3. Hosono, R. et al. J. Neurochem. 58, 1517-1525 (1992). D. J. J. molec. Biol. 215, 403-410 (1990).

5. Aalto, M. K., Keränen, S. \& Ronne, H. Cell68, 181-182 (1992).
}

uct has significant similarity to the yeast Sec1 protein (see figure). The program identified nine regions of homology throughout the protein, and estimated the probability of finding the first six of these by chance as $3.3 \times 10^{-15}$. Sec1 protein is required for the fusion of Golgi-derived vesicles with the plasma membrane. It is related to two other yeast proteins, Sly1 and Slp1, which are involved in other steps of the secretory pathway ${ }^{5}$; however, unc-18 is much more similar to Sec1 than to either of these others (as judged from the BLAST scores). I suggest that the unc- 18 product has a role analogous to that of Sec1, and promotes the fusion of synaptic vesicles with the plasma membrane. It is not clear whether its function is restricted to nerve cells, or to a subset of them, or is more general. The viability of the $u n c-18$ mutants suggests that the protein is not generally required for secretion, but it is conceivable that the existing unc-18 alleles retain a low level of activity which is sufficient for growth. Alternatively, unc-18 may belong to a family of genes whose products differ in their tissue distributions.

Hugh R. B. Pelham

MRC Laboratory of Molecular Biology,

Hills Road,

Cambridge CB2 2QH, UK

\section{Function of vertebrate $T$ gene}

SIR - Formation of mesoderm and, in particular, of notochord is a crucial event in vertebrate development. Significant advances in the understanding of notochord development in vertebrates have followed the cloning of the mouse Brachyury (T) gene ${ }^{1-4}$. Loss of function of the gene leads to a disturbance in development of the primitive streak, the most strongly affected region being the notochord ${ }^{4}$. Homologues of the mouse $T$ gene have been isolated from Xenopus ${ }^{5}$ and zebrafish ${ }^{6}$. The regions exhibiting the $T$-gene expression, however, are not always restricted to the predicted notochord territory of the embryos ${ }^{2,5,6}$. Significant roles of the $T$ gene in formation of mesoderm have been suggested, 